\title{
Imaging the root-rhizosphere interface using micro computed tomography: quantifying void ratio and root volume ratio profiles
}

\author{
Tomás Lascurain ${ }^{1}$, Vasileios Angelidakis ${ }^{1}$, Saimir Luli $^{2}$, and Sadegh $\mathrm{Nadimi}^{1, *}$ \\ ${ }^{1}$ School of Engineering, Newcastle University, United Kingdom \\ ${ }^{2}$ Preclinical In Vivo Imaging Facility, Faculty of Medical Sciences, Newcastle University, United Kingdom
}

\begin{abstract}
Root growth alters soil fabric and consequently its mechanical and physical properties. Recent studies show that roots induce compaction of soil in their immediate vicinity, a region that is central for plant health. However, high quality quantification of root influence on the soil fabric, able to inform computational models is lacking from the literature. This study quantifies the relationship between soil physical characteristics and root growth, giving special emphasis on how roots in early stage formation influence the physical architecture of the surrounding soil structure. High-resolution X-ray micro-Computed Tomography $(\mu \mathrm{CT})$ is used to acquire three dimensional images of two homogeneously-packed samples. It is observed that the void ratio profile extending from the soil-root interface into the bulk soil is altered by root growth. The roots considerably modify the immediate soil physical characteristics by creating micro cracks at the soil-root interface and by increasing void ratio. This paper presents the mechanisms that led to the observed structure as well as some of the implications that it has in such a dynamic zone.
\end{abstract}

\section{Introduction}

Plants are used as a natural solution against erosion and shallow landslides. The importance of vegetation for preventing and controlling such geotechnical events has been demonstrated and practised throughout the world. However, soil-root interaction involves multi-scale and multiphysics phenomena which need to be quantified at different scales in order to robustly predict changes in soil physical and mechanical properties [1-5].

Over the last few decades, there has been particular interest in understanding the physical interplay between soil and roots. First, a better understanding of the soil structure can result in better growth and stabilisation of plants. Soil structure changes, such as particle organisation and increases in bulk density can strongly affect the growth and diameter of roots [6]. Roots can also cause changes in soil structure [7]. A recent study by Lucas et al. [8] suggested that roots compact the soil, if the initial soil fabric does not offer a sufficient pore volume. On the other hand, if the soil consists of highly porous rhizosphere, the root uses this existing pore space. They also reported that in some cases fine root grew into more dense area. This behaviour is probably triggered by secondary factors, such as water or nutrient availability.

In this paper, two samples, one of fine and one of coarse glass ballotini are used to observe the root behaviour and quantify void ratio and root volume ratio profiles. The idealised experiments in this paper provide validation case studies for future numerical simulations.

*e-mail: sadegh.nadimi-shahraki@ncl.ac.uk

A video is available at https://doi.org/10.48448/sjwf-k771

\section{Materials and Methods}

\subsection{Sample preparation}

Two cylindrical containers with dimension of $40 \mathrm{~mm}$ height, $21 \mathrm{~mm}$ inner diameter, $1 \mathrm{~mm}$ wall thickness, made of poly(methyl methacrylate) (PMMA) were filled with two different particulate materials, i.e. spherical fine hollow glass ballotini (FGB) with $d=50 \pm 30 \mu \mathrm{m}$ and spherical coarse solid glass ballotini (CGB) with $d=1000 \pm$ $20 \mu \mathrm{m}$, using air pluviation [9]. The material properties are presented in Table 1. The procedure generates homogeneous packings. A lentil seed was then placed at a shallow depth, near the top surface of each sample (one seed per test). The samples were watered during the planting. No additional water was added during the experiments. Seed germination was initiated $24 \mathrm{hrs}$ before the planting. X-ray micro-computed tomography $(\mu \mathrm{CT})$ was adopted to investigate the spatial distributions of voids and root volume ratios for the two samples, after 7 days of growth at room temperature.

\subsection{Acquisition of X-ray computed tomography images}

$\mu \mathrm{CT}$ is a non-invasive and non-destructive imaging technique, used to visualise the inner structure of materials. The $\mu C T$ images used in this study were obtained using the Preclinical In Vivo Imaging Facility at Newcastle University, in the United Kingdom. The samples were scanned using a SkyScan $1176 \mu \mathrm{CT}$ system with a micro-focus Xray tube, using a voltage of $80 \mathrm{kV}$ and a current of $313 \mu \mathrm{A}$. 
Table 1. Material properties used in this study

\begin{tabular}{|c|c|c|c|}
\hline & $\begin{array}{l}\mathrm{d}_{50} \\
\mu \mathrm{m}\end{array}$ & $\begin{array}{l}\text { Density } \\
\mathrm{g} / \mathrm{cm}^{3}\end{array}$ & $\begin{array}{l}\text { Compressive } \\
\mathrm{MPa}\end{array}$ \\
\hline FGB & 50 & $0.15-0.70$ & $2-124$ \\
\hline CGB & 1000 & 1.53 & 65000 \\
\hline
\end{tabular}

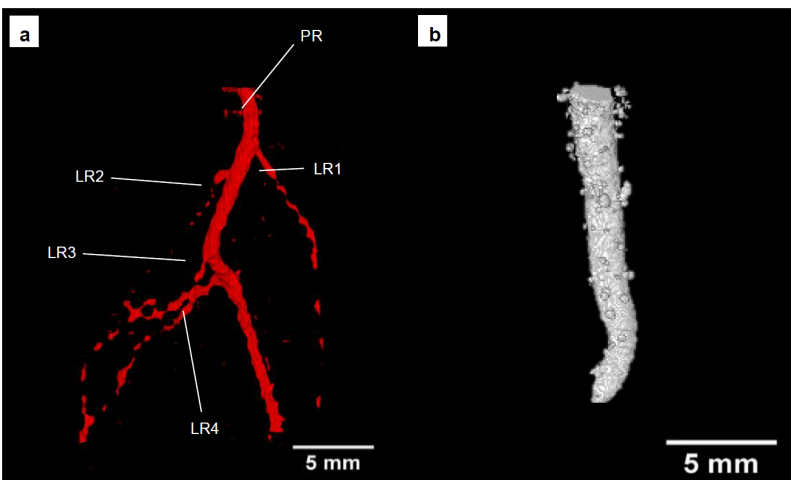

Figure 1. 3D reconstruction of the segmented root system of (a) the coarse glass beads sample with each type of root indicated (PR, primary root; LR1, lateral root 1; LR2, lateral root 2; LR3, lateral root 3; LR4, lateral root 4) and (b) the fine glass beads sample.

Cross-sectional slices were generated from the projections using the reconstruction algorithm of Feldkamp and Jesion [10] to obtain 3D images. Regarding resolution, the reconstructed images have a voxel size of $17.64 \mu \mathrm{m}$. Therefore, coarse particles are represented by more than 56 voxels across their diameter, while, fine particles are constituted by 3 voxels across their diameters.

\subsection{Image processing}

Prior to image analysis, the regions of interest (i.e. root, soil, and voids) were detected and labeled, to quantify the spatial distribution of voids and roots. This was achieved through segmentation. Segmentation was carried out semi-automatically, using the open-source software project Trainable Weka Segmentation (TWS) [11], which is a plugin of the image processing toolkit Fiji (a distribution of ImageJ) [12], combined with the machine learning toolkit Waikato Environment for Knowledge Analysis (WEKA) [13]. To segment the 3D images, a set of input pixels were manually classified as belonging to three classes, namely "root", "soil" or "void", on 10-20\% of images. Once the classifier was trained, it was used to classify the rest of the input pixels. Default image settings included in TWS were used to segment the images. Figure 1 shows the 3D reconstruction of the root system in both samples, including an identification of the primary root (PR) and lateral roots (LR) for the CGB sample.

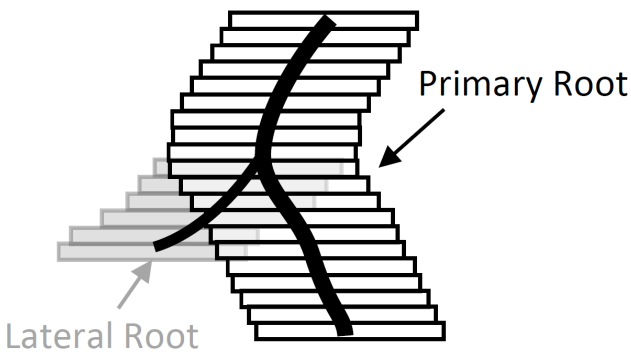

Figure 2. Schematic of laminar cylinder used as region of interest to estimate the local void ratio.

\subsection{Quantification of global and local void ratios}

Measurements of the void ratio (i.e. the volume of voids over the volume of solids) extending from the root into the bulk soil were taken, allowing for a distinction between the zone adjacent to the root surface and the zones at slightly larger distances. The regions of interest are laminar cylinders in shape, aligned with the roots as illustrated in Figure 2. The void ratio was quantified at four radii from the centroid of the root (i.e. $0.88 \mathrm{~mm}, 1.42 \mathrm{~mm}, 1.97 \mathrm{~mm}$ and $10 \mathrm{~mm}$ ), representing both local and global void ratios, and was calculated as the number of voxels corresponding to voids over the number of voxels corresponding the solids. The same approach was applied to both FGB and CGB samples.

\subsection{Quantification of root volume ratio}

The root volume ratio, defined as the total volume of roots per unit volume of soil, was calculated as the the number of voxels representing roots to the number of voxels representing solids, for each region of interest.

$$
R_{v}=\frac{\sum \text { voxel }_{\text {root }}}{\sum \text { voxel }_{\text {solid }}}
$$

This parameter is used in the soil water retention curve model proposed by $\mathrm{Ng}$ et al. [14], as the defining property of the vegetation in the shape of the curve.

\section{Results and Discussion}

Figures 3(a) and 3(b) show the void ratio profiles of the CGB and FGB samples, respectively. A general descending trend of the void ratio can be seen when moving away from the root and towards the external boundary of the container (i.e. the bulk soil), for both samples. The CGB sample shows surprisingly large local void ratio close to the root system. In the CGB sample, a decrease of void ratio with depth is also evident. However, in the FGB one, the maximum local void ratio was observed at $5 \mathrm{~mm}$ depth. This can be attributed to the formation of microcracks around the root due to capillarity effects (Figure 6(a)). The formation of these cracks is expected to have an important effect in hydraulic conductivity. In particular, these cracks reduce water capillary potential of the 


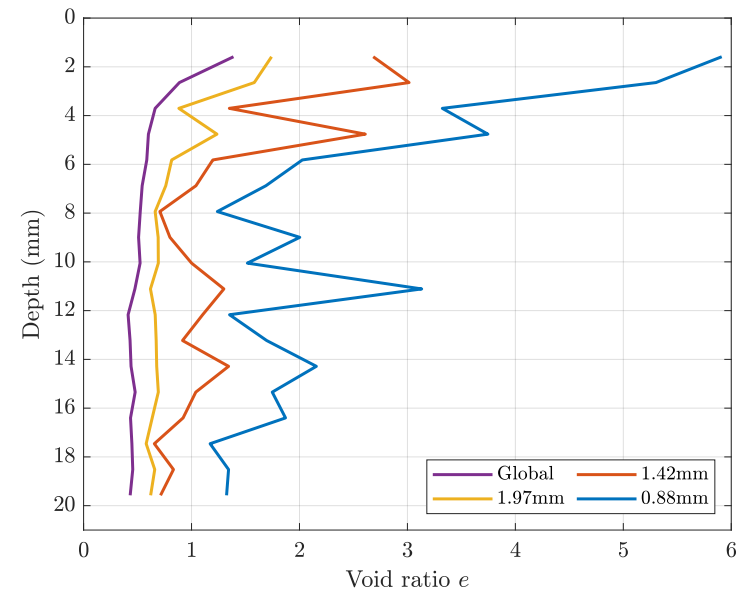

(a)

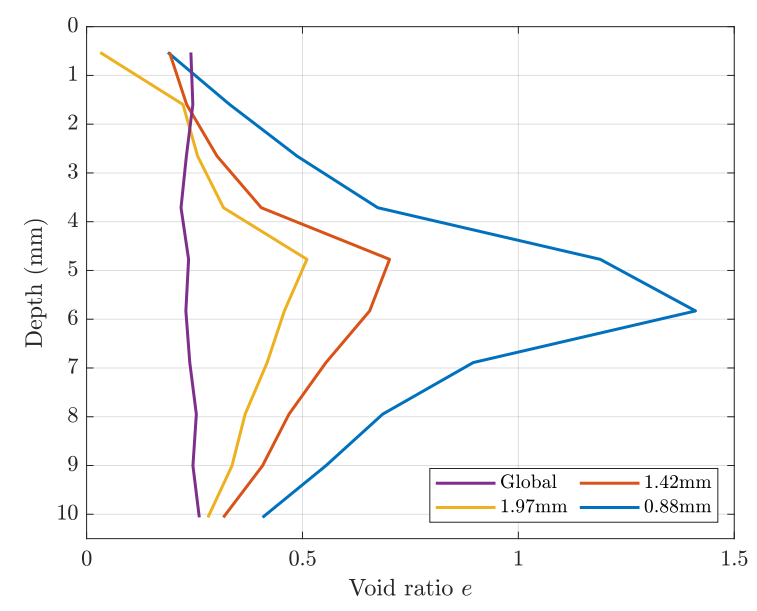

(b)

Figure 3. Void ratio profile estimated at different radii from the root centroid for (a) coarse and (b) fine glass ballotini.

surrounding particles, but maintain the pore connectivity within the rhizosphere and improve oxygen and carbon dioxide movement to and from the root system [15]. The FGB sample is more susceptible to cracking, as the beads are within the silt size range and have lower particle density. Therefore, the attractive forces between particles may play a role in the formation of microcracks.

The area surrounding the lateral roots was analysed and the void ratio profiles are presented in Figure 4. It can be seen that the void ratio of the lateral roots is slightly lower than the one of the primary root. The starting depth for each lateral root varies and represents the actual depth where the lateral root branched out. In the FGB sample, lateral roots did not grow.

Both samples exhibit a general decrease in the root volume ratio for increasing depth (Figure 5). The CGB sample shows a steeper increase with depth due to spatial variability in the lateral root growth. The fine glass beads sample, however, shows a steady decrease in root volume ratio, since the root system consists of one single root.

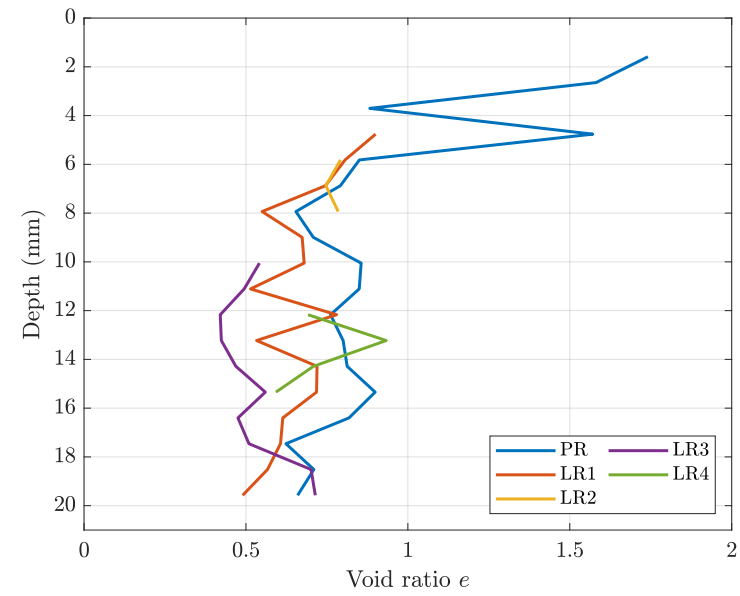

Figure 4. Void ratio profile for primary root (PR), lateral roots (LR) in coarse glass ballotini.

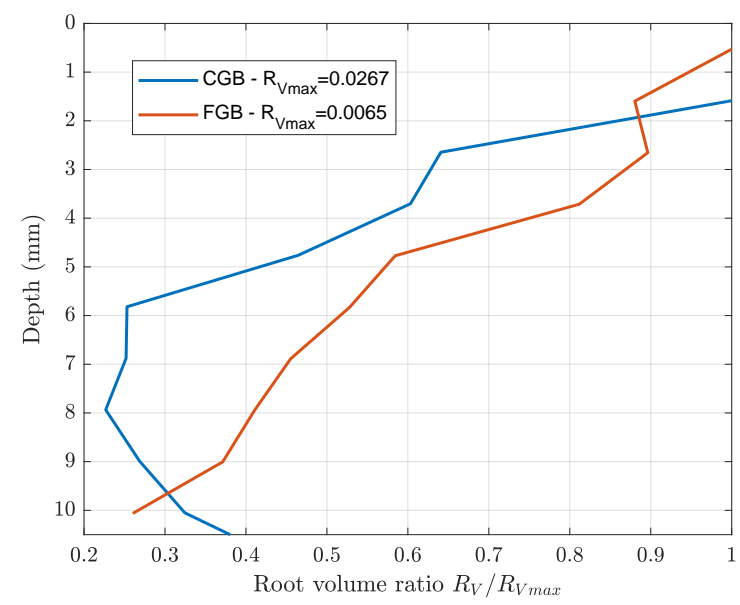

Figure 5. Normalised root volume ratio profile for coarse and fine glass ballotini.

\section{Conclusion}

3D images obtained with X-ray $\mu \mathrm{CT}$ of coarse glass ballotini and fine glass ballotini were used to quantify the spatial void ratio and root volume ratio profiles. A relatively high void ratio at the immediate vicinity of the root surface was observed after seven days of growth. The coarse sample presented a higher void ratio at the soil-root interface than the fine sample. High-resolution images revealed significant microcracks radiating around roots in the fine sample. This can be attributed to the size and density of the fine beads.

The pore structure at the root surface is also attributable to the nature of the soil and to the substances secreted by the root. The well-connected macropore found by the root facilitated the displacement of the particles creating this porous rhizosphere and enhanced physical conditions for root penetration.

The methodology proposed in this study will be extended to investigate the root growth at varying time increments and provide validation case studies for numerical simulations, which in turn will result in the improvement of conceptual soil-root interaction models. In addition to 


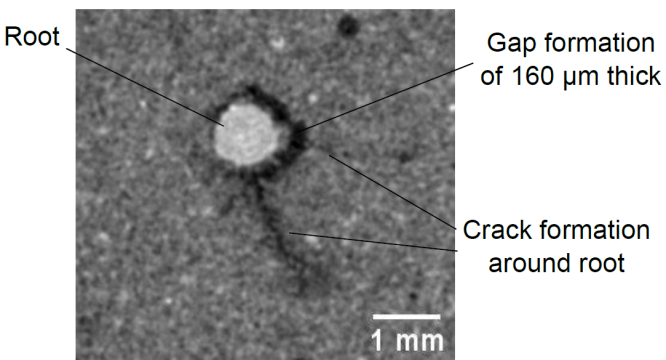

(a)

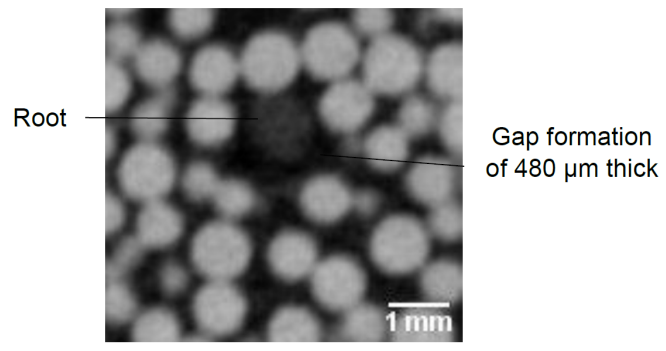

(b)

Figure 6. Greyscale images of the soil samples illustrating the soil structure after 7 days of growth. A crack formation radiating from the root surface can be observed for the (a) fine and (b) coarse glass ballotini.

improving geotechnical design, studying this dynamic interface is central for nutrient acquisition, water uptake and gaseous exchange.

\section{References}

[1] E. Kolb, V. Legué, M.B. Bogeat-Triboulot, Physical root-soil interactions. Phys. Biol. 14(6), 065004 (2017)

[2] J.A. Smethurst, D. Clarke, W. Powrie, Factors controlling the seasonal variation in soil water content and pore water pressures within a lightly vegetated clay slope. Géotechnique 62(5), 429-446 (2012)

[3] C.W.W. Ng, A.K. Leung, K.X. Woon, Effects of soil density on grass-induced suction distributions in compacted soil subjected to rainfall. Can. Geotech. J. 51(3), 311-321 (2014)

[4] A. Fraccica, E.E. Romero Morales, T. Fourcaud, Multi-scale effects on the hydraulic behaviour of a root-permeated and compacted soil. In IS-Glasgow 2019-7th International Symposium on Deformation Characteristics of Geomaterials (EDP Sciences, 2019)
[5] A.K. Leung, A. Garg, C.W.W. Ng, Effects of plant roots on soil-water retention and induced suction in vegetated soil. Eng. Geol. 193, 183-197 (2015)

[6] J.E. Aravena, M. Berli, T.A. Ghezzehei, S.W. Tyler, Effects of root-induced compaction on rhizosphere hydraulic properties-X-ray microtomography imaging and numerical simulations. Environ. Sci. Technol. 45, 425-431 (2011)

[7] F. Anselmucci, E. Andó, L. Sibille, N. Lenoir, R. Peyroux, C. Arson, G. Viggiani, A.G. Bengough, Rootreinforced sand: kinematic response of the soil. In 7 th International Symposium on Deformation Characteristics of Geomaterials, IS-Glasgow (EDP Sciences, 2019)

[8] M. Lucas, S. Schlüter, H.J. Vogel, D. Vetterlein, Roots compact the surrounding soil depending on the structures they encounter. Sci. Rep. 9(1), 1-13 (2019)

[9] S. Nadimi, J. Fonseca, Enhancing soil sample preparation by thermal cycling. Géotechnique 66(11), 953-958 (2016)

[10] L.A. Feldkamp, G. Jesion, Chapter 2: Inversion, Imaging and Reconstruction, In Review of Progress in Quantitative Nondestructive Evaluation. SpringerVerlag US 555-566 (1986)

[11] I. Arganda-Carreras, V. Kaynig, C. Rueden, K.W. Eliceiri, J. Schindelin, A. Cardona, H. Sebastian Seung, Trainable Weka Segmentation: a machine learning tool for microscopy pixel classification. Bioinformatics 33(15), 2424-2426 (2017)

[12] J. Schindelin, I. Arganda-Carreras, E. Frise, V. Kaynig, M. Longair, T. Pietzsch, S. Preibisch, C. Rueden, S. Saalfeld, B. Schmid, J.Y. Tinevez, Fiji: an open-source platform for biological-image analysis. Nat. methods 9(7), 676-682 (2012)

[13] M. Hall, E. Frank, G. Holmes, B. Pfahringer, P. Reutemann, I.H. Witten, The WEKA data mining software: an update. ACM SIGKDD explorations newsletter 11(1), 10-18 (2009)

[14] C.W.W. Ng, J.J. Ni, A.K. Leung, Z.J. Wang, A new and simple water retention model for root-permeated soils. Geotech. Lett. 6(1), 106-111 (2016)

[15] J.R. Helliwell, A.J. Miller, W.R. Whalley, S.J. Mooney, C.J. Sturrock, Quantifying the impact of microbes on soil structural development and behaviour in wet soils. Soil Biol. Biochem. 74, 138-147 (2014) 